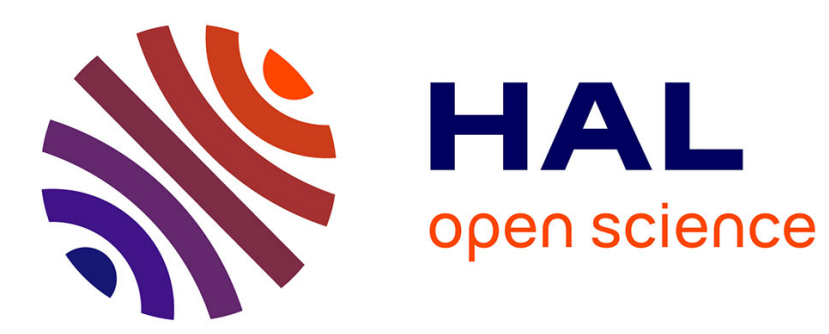

\title{
Dynamics of sensory processing in the dual olfactory pathway of the honeybee
}

\author{
Martin Nawrot
}

\section{To cite this version:}

Martin Nawrot. Dynamics of sensory processing in the dual olfactory pathway of the honeybee. Apidologie, 2012, 43 (3), pp.269-291. 10.1007/s13592-012-0131-3 . hal-01003653

\section{HAL Id: hal-01003653 https://hal.science/hal-01003653}

Submitted on 1 Jan 2012

HAL is a multi-disciplinary open access archive for the deposit and dissemination of scientific research documents, whether they are published or not. The documents may come from teaching and research institutions in France or abroad, or from public or private research centers.
L'archive ouverte pluridisciplinaire HAL, est destinée au dépôt et à la diffusion de documents scientifiques de niveau recherche, publiés ou non, émanant des établissements d'enseignement et de recherche français ou étrangers, des laboratoires publics ou privés. 


\title{
Dynamics of sensory processing in the dual olfactory pathway of the honeybee
}

\author{
Martin Paul NAwrot ${ }^{1,2}$ \\ ${ }^{1}$ Neuroinformatik/Theoretische Neurobiologie, Institut für Biologie, Freie Universität Berlin \\ Königin-Luise-Straße 1-3, 14195 Berlin, Germany \\ ${ }^{2}$ Bernstein Center for Computational Neuroscience Berlin, Germany
}

Received 19 November 2011 - Revised 22 January 2012 - Accepted 20 February 2012

\begin{abstract}
Insects identify and evaluate behaviorally relevant odorants in complex natural scenes where odor concentrations and mixture composition can change rapidly. This requires fast and reliable information processing in the olfactory system. Here, we review recent experimental findings and theoretical hypotheses on olfactory processing in the honeybee with a focus on its temporal dynamics. Specifically we address odor response characteristics of antennal lobe interneurons and projection neurons, local processing of elemental odors and odor blends, the functional role of the dual olfactory pathway in the honeybee, population coding in uniglomerular projection neurons, and a novel model for sparse and reliable coding in projection neurons and mushroom body Kenyon cells. It is concluded that the olfactory system of the honeybee implements a fast and reliable coding scheme optimized for processing dynamic input within the behaviorally relevant temporal range.
\end{abstract}

antennal lobe / sparse code / latency code / odor trace / olfaction

\section{INTRODUCTION}

In their natural environment, insects sense and evaluate blends of olfactory stimuli in the context of a complex background. In the honeybee, behaviorally relevant odorants range from simple odors composed of single or few chemical compounds to highly complex odor blends, e.g., pheromones, food signals, and flower odors. Due to the turbulent and discontinuous nature of odor plumes, airflow, and animal velocity, the composition and concentration of odor blends fluctuate

Electronic supplementary material The online version of this article (doi:10.1007/s13592-012-0131-3) contains supplementary material, which is available to authorized users.

Corresponding author: M.P. Nawrot, martin.nawrot@fu-berlin.de Manuscript editor: Bernd Grünewald on various time scales, including highly rapid changes (e.g., Riffell et al. 2008; Vickers et al. 2001; for review, see Martin et al. 2011).

Behavioral experiments showed that honeybees discriminate odor concentrations (Pelz et al. 1997; Wright et al. 2005) and that they distinguish between odor mixtures (Chandra and Smith 1998). More recently, it has been shown that bees can learn to discriminate odors that are presented for only a very short period of 200 ms (Wright et al. 2009; Fernandez et al. 2009). Longer stimuli may be required to reach the same learning rate and performance in odor discrimination under challenging conditions such as low odor concentrations (Wright et al. 2009) or when discriminating different mixture ratios (Fernandez et al. 2009). Recordings from the mushroom body output neurons of honeybees during and after odor conditioning showed that their population reliably encodes the learned value 
of an odor within less than $150 \mathrm{~ms}$ (Strube-Bloss et al. 2011). A similar speed of discriminating dissimilar odors (Abraham et al. 2004), different mixture ratios of a binary mixture (Uchida and Mainen 2003), or novel odors from learned odors (Wesson et al. 2008) has been demonstrated in behavioral experiments with rodents.

How does the sensory network in the honeybee achieve a fast and reliable processing of the olfactory input that allows the animal to track changes in its environment and to rapidly form odor percepts as, e.g., required during a memory retention test? An increased number of experimental studies have provided us with an improved understanding of odor processing at different stages of the olfactory pathway. In the present review, we focus on the temporal dynamics of information processing at the level of single neurons and neural populations as monitored in electrophysiological experiments, and we present recent theoretical considerations that provide hypotheses for a functional role of the dual odor pathway and for reliable and sparse coding in the sensory network.

\section{THE OLFACTORY PATHWAY OF THE HONEYBEE}

The peripheral stage of the olfactory system in the honeybee comprises $\sim 60,000$ olfactory sensory neurons (OSNs; Esslen and Kaissling 1976), which are located predominantly in pore plate sensillae on the antennae. The antennal lobe (AL) represents the first stage of olfactory processing. It is composed of $\sim 160$ glomeruli which represent the synaptic sites between OSNs, local interneurons (LNs), and the projection neurons (PNs). LNs are morphologically restricted to the antennal lobe and innervate multiple glomeruli. PNs form the output of the antennal lobe sending their axons to central brain structures (Figure 1). Hymenoptera in general and honeybees in particular feature an anatomical specialty, the dual olfactory pathway. Each OSN conveys chemosensory information through one of the four antennal tracts (T1-T4, Figure 1a, Abel et al. 2001). Approximately 920 uniglomerular PNs (Rybak
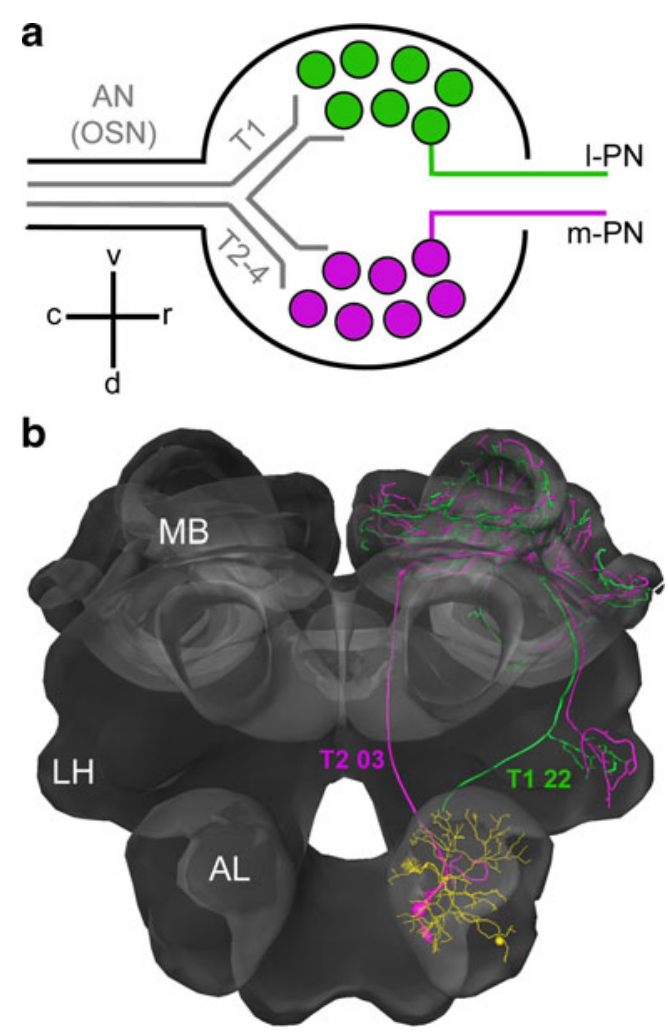

Figure 1. Dual olfactory pathway in the honeybee. a Antennal lobe $(A L)$ circuitry. OSN axons innervate the AL through four antennal nerve $(A N)$ tracts T1T4. Uniglomerular 1-PNs exclusively innervate glomeruli that receive input through $\mathrm{T} 1$, uniglomerular $\mathrm{m}-\mathrm{PNs}$ innervate glomeruli that receive input through the antennal tracts T2-T4. For the brain, the axis rostral-caudal refers to the neural axis rather than to the body axis, adopting the convention by Kirschner et al. (2006). Accordingly, glomeruli innervated by the $\mathrm{T} 1$ tract are located ventrally, and T2-T4 are situated dorsally with respect to the neural axis. b Morphological reconstructions of one 1-PN innervating the glomerulus T1-22 (green) and one m-PN innervating the glomerulus T2-03 (magenta) demonstrate their separate projections along the lateral and the median APT to the mushroom body $(M B)$ lip regions and to the lateral horn $(L H)$. The $\mathrm{LN}$ (yellow) innervates large parts of the AL homogenously. Staining and reconstruction by S Krofczik. We thank J Rybak for 3D registration and visualization in the honeybee standard brain (Rybak et al. 2010; Rybak 2012), which can be accessed in the supplementary material. 
2012; Kirschner et al. 2006) divide into lateral PNs (l-PNs) and median PNs (m-PNs). The former receive input almost exclusively from $\mathrm{T} 1$ glomeruli (Figure 1a, green circles) and send their axons along the lateral antenno-protocerebral tract (APT) to higher-order brain centers, first to the lateral horn (LH) and then to the mushroom body (MB). The median PNs (mPNs) exclusively originate in T2-T4 glomeruli (Figure 1a, magenta circles) and project along the median APT, first to the MB and then to the LH (Abel et al. 2001; Kirschner et al. 2006; Rössler and Zube 2011). The third group of multiglomerular PNs branch in several glomeruli, and their projections travel along distinct tracts mainly to the protocerebrum (for review, see Galizia and Rössler 2010).

While the basic anatomy of the olfactory system is conserved across different insect species, there are also marked inter-species differences (for review, see Martin et al. 2011). For instance, the dual olfactory pathway of the honeybee where uniglomerular 1-PNs and mPNs receive exclusive input from only one of the AL hemilobes is specific for Hymenoptera (Rössler and Zube 2011). The division into a lateral and a median fiber tract exists in, e.g., bees and ants (Hymenoptera), flies (Diptera), cockroaches (Blattaria), and moths (Lepidoptera) but not in the locust (Orthoptera) (for review, see Galizia and Rössler 2010). Clear differences are also observed in the structure of the local interneuron network of the AL. In the locust, for example, it comprises only about 300 inhibitory LNs that do not produce sodium action potentials (Laurent and Davidowitz 1994). In comparison, the honeybee AL network is composed of $\sim 4,000-5,000$ spiking interneurons (Fonta et al. 1993; Abel et al. 2001). Excitatory interneurons were found in Drosophila (Shang et al. 2007), but it is not yet known whether they also exist in the honeybee and other insects. These evolutionary differences likely reflect functional specializations, and they may imply that different mechanisms of input processing as well as different coding strategies are employed to meet specific behavioral requirements (Martin et al. 2011).

\section{LOCAL PROCESSING IN THE HONEYBEE ANTENNAL LOBE NETWORK}

The local interneuron network of the AL processes the OSN input transforming it into the AL output code at the level of the PN population. Our present knowledge on AL processing in the honeybee to a large degree is based on Ca-imaging experiments that studied glomerular response patterns across a number of simultaneously imaged glomeruli focusing on either the AL input (composite signal resulting from bath application of the dye) or the PN output (retrograde staining of PNs) (e.g., Joerges et al. 1997; Galizia and Menzel 2000; Sachse and Galizia 2002; Deisig et al. 2006, 2010; Sandoz 2011). Here we focus on results obtained by means of intracellular recording and staining of individual AL neurons (Fig 1). Two major drawbacks of Ca-imaging experiments make it necessary to perform complementary studies of AL processing at the level of single neurons. First, the Ca signal is comparably slow and, thus, fast temporal dynamics on time scales of $\sim 1-100 \mathrm{~ms}$ are difficult to address. Second, $\mathrm{Ca}$ imaging from the AL in the bee has thus far only been achieved from superficially located glomeruli, which represent glomeruli of the lateral APT. This limitation might be partly overcome by means of two-photon imaging techniques that also allow monitoring of glomerular activity in the tracts T2-T4 (Haase et al. 2010). An important methodological advancement achieved imaging from uniglomerular 1$\mathrm{PN}$ and $\mathrm{m}-\mathrm{PN}$ boutons in the MB lip region (Yamagata et al. 2009).

\subsection{Different response characteristics of local interneurons and projection neurons}

We distinguish two major morphological classes of AL neurons, LNs and PNs. What is known about their characteristic response properties? A recent metastudy (Meyer et al. 2011; Meyer 2011) combined three independent data sets of intracellular recordings from AL neurons 
(Galizia and Kimmerle 2004; Krofczik et al. 2008; Meyer 2011) in order to relate response characteristic of single neuron spike trains to the two distinct morphological classes of LNs and PNs. Single neuron responses during the first $800 \mathrm{~ms}$ of odor stimulation were characterized by different features, which allowed their classification as either LN or PN with $81 \%$ accuracy in 33 morphologically identified neurons. Applying this classification model to a total of 80 neurons showed significant classrelated differences in several features. Figure 2e summarizes the results for three relevant features that permit a direct interpretation. One important difference was found for the average response latency (i.e., the time from odor a
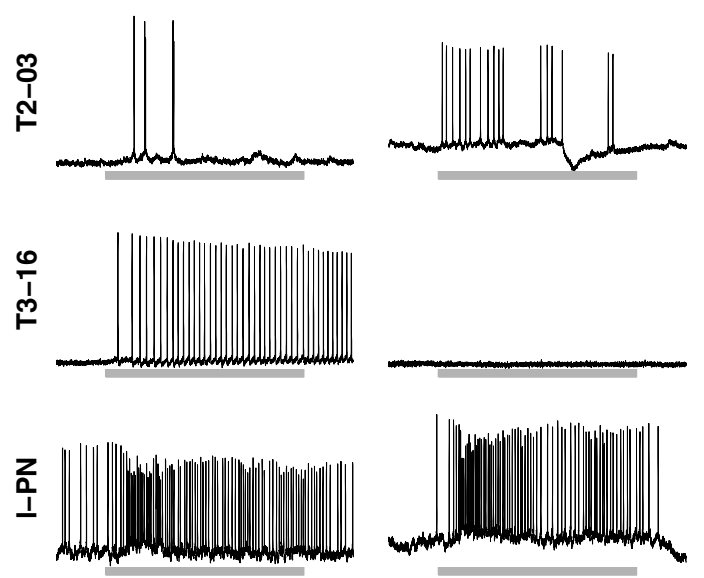

nonanol
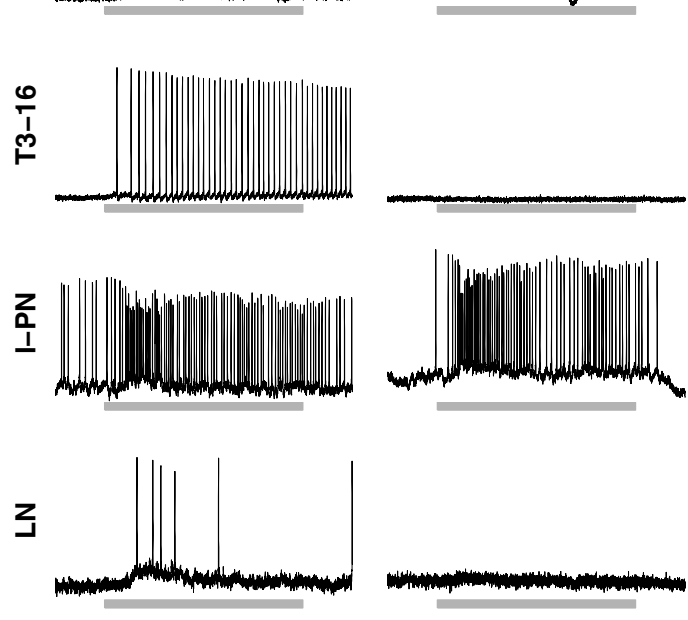

b
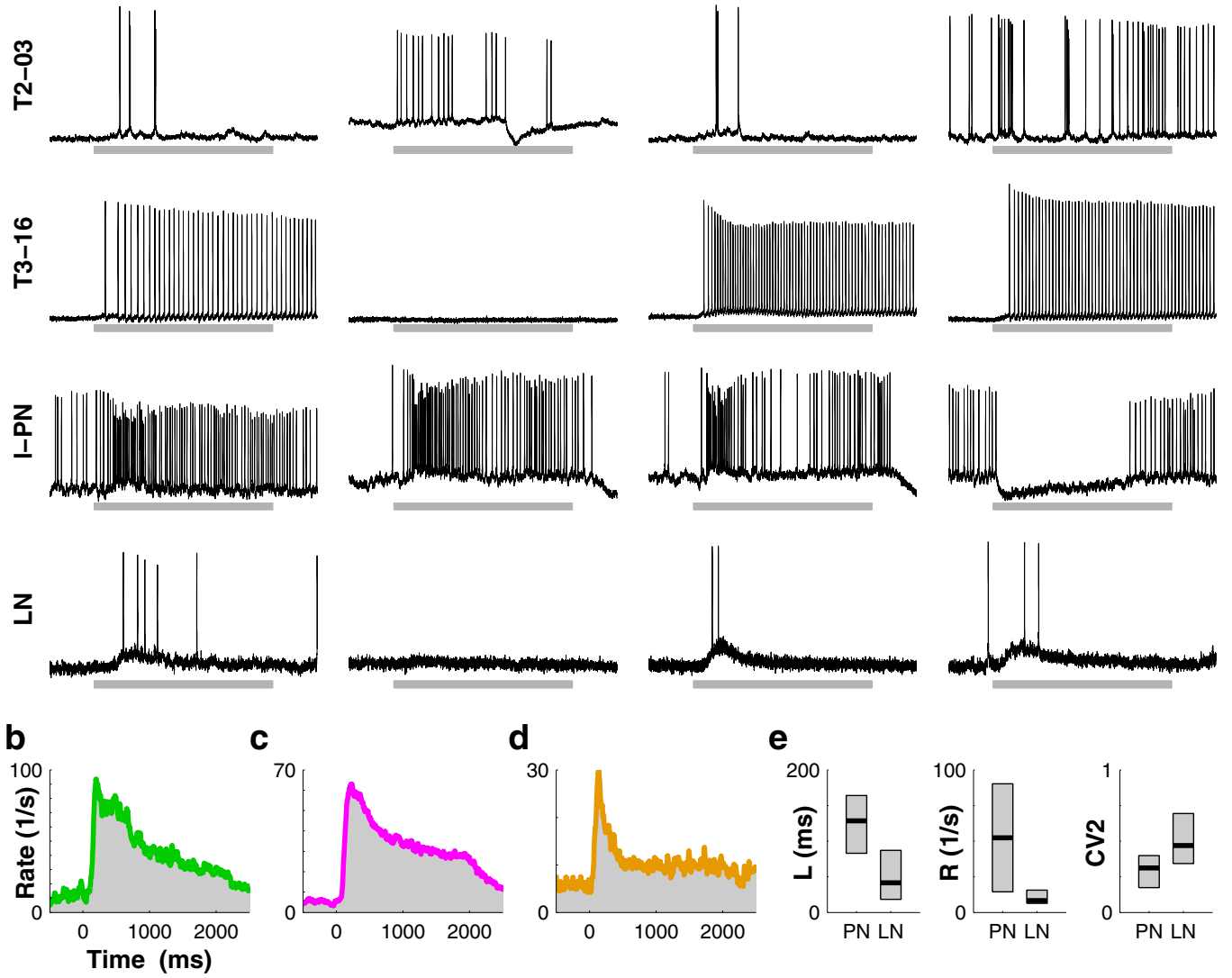

Figure 2. Intracellular recordings of antennal lobe neurons. a Four single neuron responses to three single compound odors as indicated and their tertiary mixture (mix). The top two neurons were morphologically identified as uniglomerular m-PNs and glomeruli are indicated. The neuron displayed in the third row was morphologically identified as uniglomerular 1-PN; innervated glomerulus could not be identified. Lower neuron was identified as LN. Horizontal gray bars indicate stimulus period of $2 \mathrm{~s}$ duration. b-d Average firing rate profiles of 1-PNs (b), m-PNs (c), and LNs (d). e Median and quartiles for response latencies $L$, response rate $R$, and CV2 for neurons classified as LNs and PNs. The CV2 quantifies interval variability. For each pair of neighboring inter-spike intervals, it measures their standard deviation divided by their mean before averaging across all interval pairs. High values of CV2 indicate irregular spiking; low values signify regular spiking. a-d Reproduced from data set in Krofczik et al. (2008). e Reproduced from Meyer et al. (2011). 
stimulation at the antennal site to the neuronal response onset), which was significantly shorter in LNs (median $42 \mathrm{~ms}$ ) than in PNs (median $\sim 129 \mathrm{~ms}$, Figure 2e), confirming an earlier observation (Krofczik et al. 2008). A similar difference in response latency for LNs and PNs has been reported for the moth in a Ca-imaging study (Fujiwara et al. 2009). Kuebler et al. (2011) reported shorter LN response in moth specifically during mixture presentation. A second important feature was the response amplitude. PNs responded with considerably higher average peak firing rates as measured from baseline (median $\sim 52 \mathrm{~Hz}$ ) than LNs (median $\sim 8 \mathrm{~Hz}$, Figure 2e). A third interesting feature relates to the spike train irregularity that was assessed by the CV2, a local measure of inter-spike interval variability (Holt et al. 1996; Ponce-Alvarez et al. 2010). PNs showed a more regular spike response pattern, indicated by a lower average CV2 of 0.32 , than LNs (CV2= 0.50 , Figure 2e).

What are the differences with respect to the odor response spectra in PNs and LNs? Here, a direct quantitative comparison between different studies is complicated by the fact that different odor spectra have been tested and different odor concentrations have been used. Moreover, individual studies typically focused on recordings of either LNs or PNs and thus within-study comparisons typically had to rely on small sample sizes for one class. Still, we can make out three general trends. First, excitatory response spectra seem broader for LNs than for PNs. The relative number of activating odors per LN lies in the range of $\sim 60-75 \%$ (Sun et al. 1993; Krofczik et al. 2008). For PNs the measured odor response spectra range from $\sim 40 \%$ to $65 \%$ (Sun et al. 1993; Müller et al. 2002; Krofczik et al. 2008; Yamagata et al. 2009). Second, PNs exhibit inhibitory responses more frequently, whereas they are rarer in LNs (Sun et al. 1993; Krofczik et al. 2008). Third, mixtures in comparison to elemental odors increased the response spectrum in LNs (Sun et al. 1993; Krofczik et al. 2008) while a suppression of excitatory responses to mixtures has been repeatedly observed in PNs of the 1-
APT indicating a more narrow tuning to mixtures at the AL output (Galizia and Kimmerle 2004; Krofczik et al. 2008; Yamagata et al. 2009).

\subsection{Characteristic differences of lateral and median uniglomerular projection neurons}

Based on intracellular recording and staining of uniglomerular projection neurons, Müller et al. (2002) reported that, in the honeybee, the segregation into the median and the lateral pathways does not reflect a general distinction between different odors or between general odors and pheromones. The authors concluded that the dual pathway likely reflects the implementation of a dual odor coding strategy rather than a separation in the olfactory chemical space, a view that has received support elsewhere (e.g., Rössler and Zube 2011; Brandstätter and Kleineidam 2011; Deisig et al. 2010; Sandoz 2011). At the more descriptive level, it appeared that $\mathrm{m}$-PNs displayed a more complex response pattern during prolonged odor stimulation than 1-PNs, which showed stereotypic phasic-tonic responses (Müller et al. 2002; cf. Figure 2a). Their finding of considerably longer response latencies in $\mathrm{m}$ PNs, however, could not be confirmed in an independent study (Krofczik et al. 2008).

Yamagata et al. (2009) performed confocal imaging of $\mathrm{Ca}$ transients of presynaptic boutons in the MB lip region of the honeybee and reported three major differences in 1-PN and m$\mathrm{PN}$ odor encoding. The first difference refers to the specificity of the odor response spectra. Tested with a set of seven different single compound odors 1-PNs showed more specific responses with an average of $3.1 \pm 0.24(\sim 44 \%)$ activating odors, while m-PNs exhibited a slightly broader odor tuning with average $4.3 \pm$ $0.37(59 \%)$ exciting odors. This is in line with results obtained in intracellular recordings where on average $\sim 45 \%$ (mixtures) to $\sim 55 \%$ (single compound odors) elicited spike responses in 1-PNs and $\sim 65 \%$ of odors activated $\mathrm{m}-\mathrm{PNs}$, irrespective of odor complexity 
(Krofczik et al. 2008). In contrast, the study by Müller et al. (2002) found that m-PNs are more specific ( $25 \%$ activating odors) than 1-PNs $(\sim 50 \%)$, albeit tested at the highest stimulus concentration (pure odors). The general result of a rather broad odor tuning at the level of PNs has been observed in different insect species (Wilson et al. 2004; Schlief and Wilson 2007; Perez-Orive et al. 2002). Noteworthy, the result of a broad odor tuning in insect PNs contrasts results in rodents where a sparse odor representation was reported in mitral cells of the olfactory bulb (Davison and Katz 2007; Rinberg et al. 2006).

The second difference refers to the concentration dependence of odor responses. In m-PN boutons, the amplitude of the $\mathrm{Ca}$ responses typically increased with odor concentration. On the contrary, 1-PNs typically responded even for very low concentrations and revealed little or even a negative relation of response strength and odor concentration. The latter effect largely depended on suppressed responses in 1-PNs at higher concentration, indicating a stronger inhibitory component in 1-APT processing (Yamagata et al. 2009). In a previous Caimaging study, Sachse and Galizia (2003) employed retrograde staining of 1-PNs, demonstrating pronounced concentration dependence in T1 glomeruli. It was hypothesized that these differing results can be explained by an inhibitory mechanism that acts on the l-PN output (Yamagata et al. 2009; Schmuker et al. 2011), e. g., through localized inhibition of the spike initiation zone, or through inhibition at the level of the calyx where PN boutons may be inhibited by GABAergic recurrent neurons (Rybak and Menzel 2010; Ganeshina and Menzel 2001).

Finally, for binary mixtures, the authors observed mixture suppression in 1-PN boutons but rarely in m-PN boutons, in line with the results from intracellular recordings that showed mixture suppression in a small sample of 1-PNs but not in a large sample of m-PNs (Krofczik et al. 2008). This finding is in agreement with earlier observations of mixture suppression of the Ca signal in T-1 glomeruli (1-APT) which is more pronounced at the level of the PN output signal than at the level of the composite signal which largely reflects OSN input (Deisig et al. 2006, 2010; Sachse and Galizia 2002; Joerges et al. 1997).

\subsection{Mixture processing at the single neuron level}

Most odorants that honeybees encounter in their natural environment are blends of many different chemical compounds. At the behavioral level, honeybees can distinguish a mixture from its individual elements (Deisig et al. 2001, 2002, 2003), and they can distinguish different mixture ratios (Fernandez et al. 2009). This requires that complex blends as well as their elemental components are perceived as individual objects. The former requires analytic processing whereby the olfactory stimulus is decomposed into its elemental components. The latter requires synthetic processing that leads to a representation of the odor blend as an entity of its own.

As mentioned above, one experimentally confirmed form of synthetic processing is expressed in mixture suppression, which increases separability between odor mixtures at the level of the AL output. This has been repeatedly observed in Ca-imaging experiments from T1 glomeruli innervated by 1-PNs (cf. Section 3.2). One example of mixture suppression is shown in Figure $2 \mathrm{a}$ where the 1-PN responds to three elemental odors but is inhibited in response to their tertiary mixture. As shown in Figure 3, the inhibition observed in the intracellular membrane potential typically shows a fast onset on the order of $\sim 60 \mathrm{~ms}$, which matches well with the shorter response latencies of LNs (Figure 2e). In contrast, mixture suppression was scarcely observed in m-PNs (Yamagata et al. 2009; Krofczik et al. 2008). Consequently, Deisig et al. (2010) formulated the hypothesis that 1-PNs provide central brain structures with non-elemental information, whereas m-PNs represent the relative quantities of elemental components.

The recent study by Meyer and Galizia (2012) investigated the detailed temporal in- 

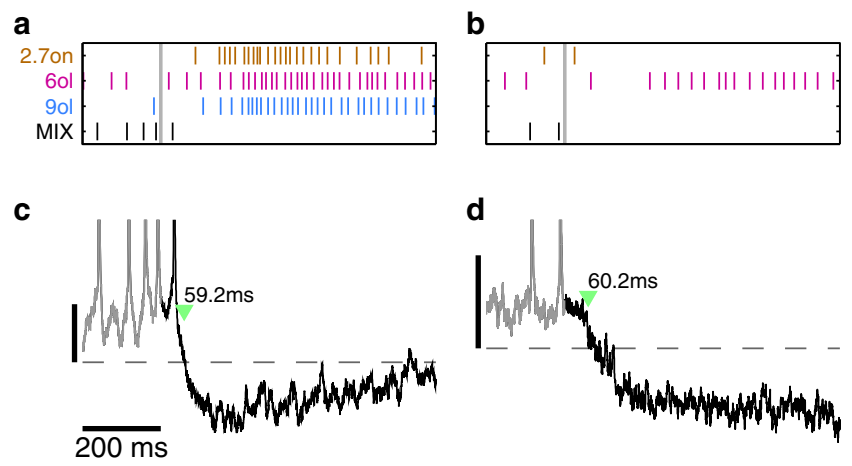

Figure 3. Mixture suppression in lateral projection neurons. a, b Response suppression in two 1-PNs. Single trial spike trains for three single compound odors and their tertiary mixture as indicated. Vertical gray bars indicate odor onset. c, d Single trial membrane potential in the same two neurons in response to the mixture shows clear inhibition. Green triangles indicate estimated onset time of inhibition. Vertical scale bars indicate $2 \mathrm{mV}$. Total stimulus duration is $2 \mathrm{~s}$. Reproduced from Krofczik et al. (2008).

volvement of single AL neurons in analytic and synthetic processing by means of intracellular recordings. The authors designed a novel paradigm testing temporally perfect and imperfect binary mixtures. Each neuron was tested with each component individually and with their synchronous presentation (perfect mixture). In addition, two imperfect mixtures were constructed by delaying the onset of either of the two components by $50 \mathrm{~ms}$, resembling the delayed encounter of odors emitted by independent odor sources. Their results showed that about half of the neurons could be classified as "elemental" responding to the perfect and imperfect mixture in the same way as to their dominant component (hypoadditive response). The other half were classified as "configural," that is, the mixture representation in those neurons obtained a new quality being different from the representation of the mixture components. Elemental neurons could be grouped into neurons with short and long response latencies, possibly relating to faster LNs and slower PNs (Figure 2e). Configural neurons displayed inbetween latencies and exhibited diverse mixture response behaviors. The authors reported an interesting tendency that showed when comparing response latencies within configural neu- rons. Their responses to imperfect mixtures were delayed with respect to the individual component responses. This might indicate comparative or competitive processing among different sensory cues that become separable due to a short temporal delay and could be important for the formation of two distinct rather than one single odor percept.

\section{POPULATION CODING IN UNIGLOMERULAR PROJECTION NEURONS}

Uniglomerular PNs form the major output of the $\mathrm{AL}$ and the sole direct olfactory input from the AL to the mushroom body. How are odors encoded in the population activity of PNs? How fast does this code emerge after the onset of a stimulus? Is there an odor trace outlasting the stimulus that could subserve Hebbian plasticity at downstream synapses?

\subsection{Fast rate coding}

On average, each PN is activated by $\sim 50 \%$ of tested odors (cf. Section 3.2). This means, in turn, that each odor activates about half of the 
total PN population. This is demonstrated in Figure $4 \mathrm{a}-\mathrm{c}$ where we reconstructed the $\mathrm{PN}$ population activity from the data set in Krofczik et al. (2008). To this end, $N=17$ single PNs

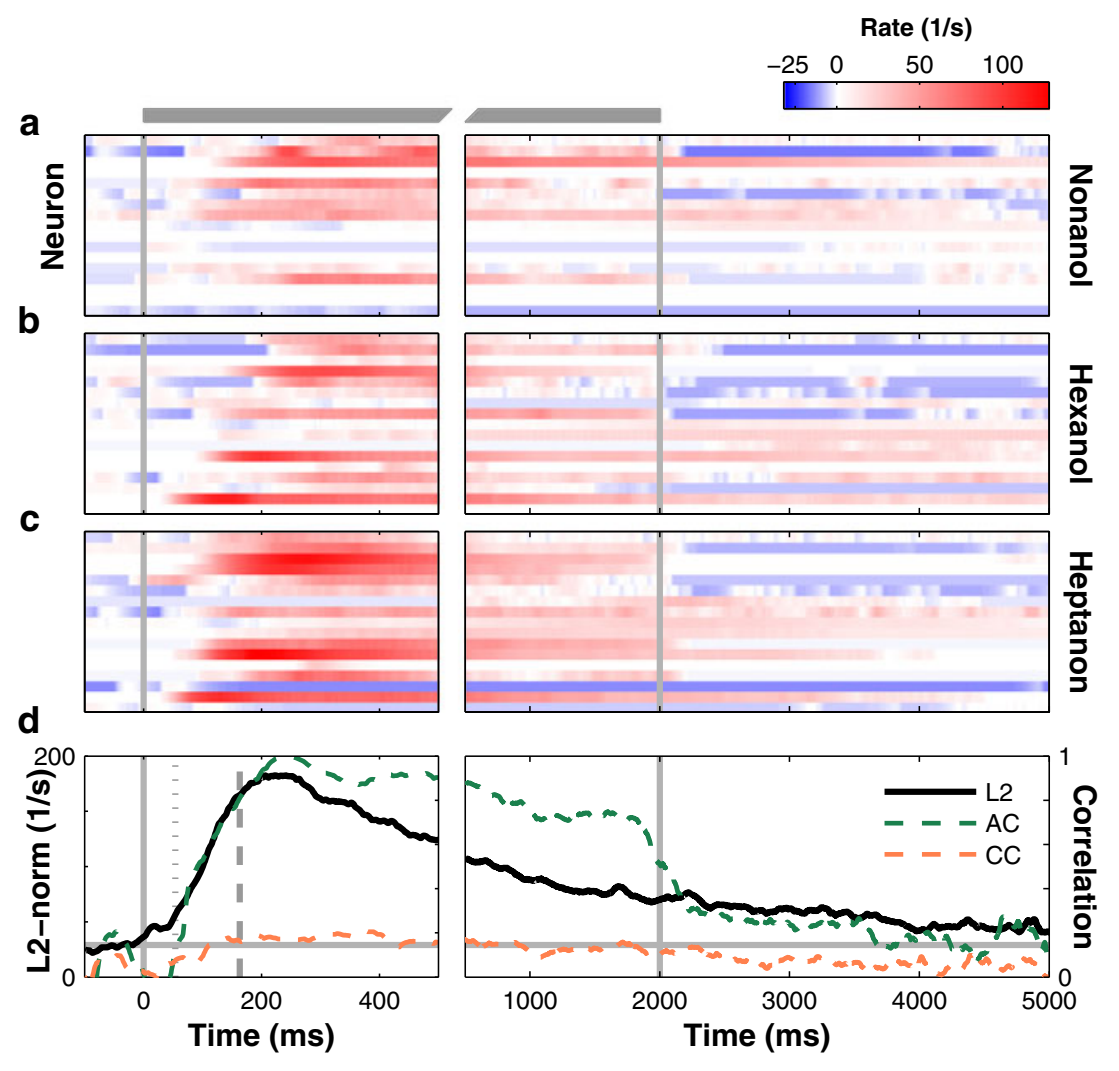

Figure 4. Rapid and sustained population coding in uniglomerular projection neurons. a-c Ensemble rate code in a pseudo-population of $N=17$ projection neurons ( $13 \mathrm{~m}$-PNs and 4 1-PNs from top $)$ in response to three different odors as indicated. For each neuron, the firing rate as a function of time is color-coded where red color signifies a rate increase from baseline (white), blue a rate decrease from baseline. Spatiotemporal activation patterns are odor specific. Left the spatial response pattern of activated and non-activated neurons develops rapidly within tens of milliseconds after odor onset (vertical gray line). Right some neurons display a continued odor response during several seconds after stimulus offset (vertical gray line) while others stop firing immediately. Trial-averaged firing rates were estimated by means of kernel convolution using the kernel function $\alpha(t)=t \times \exp (-t / \tau)$ either in causal (left, $\tau=25 \mathrm{~ms}$ ) or anti-causal (right, $\tau=50 \mathrm{~ms}$, time-flipped) fashion. Horizontal gray bar at top indicates the $2 \mathrm{~s}$ stimulus presentation. d Time-resolved Euclidean distance (L2norm, black curve) averaged across all three odor pairs. The ensemble rate code becomes rapidly significant ( $>3$ standard deviations above baseline, gray horizontal line) within $54 \mathrm{~ms}$ (dotted gray line) and reaches $90 \%$ of its peak value at $163 \mathrm{~ms}$ (dashed gray line). The Euclidean distance slowly decays as the neuronal population rate decreases. Information about odor identity is still present after stimulus offset reaching baseline approximately $3 \mathrm{~s}$ after stimulus offset. The average autocorrelation function $(A C)$ of the neuronal firing rate pattern as determined at the time of the maximal Euclidean distance with the firing pattern at later times assumes high values throughout the stimulus presentation, indicating a stable response pattern. Autocorrelation drops rapidly after odor offset, reflecting the fact that the neuronal responses remain stable only in a small subset of neurons. The average correlation across different odors (CC) maintains a low baseline level throughout the observation. Data are reproduced from Krofczik et al. (2008). 
recorded in different animals were pooled to construct the population response to three different odors. The color code represents the time-varying amplitude of the firing rates where red (blue) indicates an increase (decrease) with respect to the average baseline activity. Different odors evoked different combinatorial patterns of activated and non-activated neurons. For instance, the odor nonanol activated only a subset of neurons, while other neurons remained silent or were suppressed with respect to their spontaneous activity before odor onset. Hexanol, in comparison, activated a different but overlapping subset of neurons. Such a combinatorial odor code was first discovered in the honeybee in a series of Ca-imaging studies at the level of glomerular activation patterns (Joerges et al. 1997; Galizia et al. 1999; Galizia and Menzel 2000; Guerrieri et al. 2005).

If we consider a simple combinatorial code where odors are represented by the spatial pattern of activated and non-activated neurons with an average number $k$ of activated PNs in a total population of $N$ neurons, then it follows that we can represent the largest number of different patterns (i.e., the largest number of different odorants) if $k=N / 2$. Thus, the empiric finding that, on average, about half of the glomeruli are activated by a specific odor might indicate that local computation within the $\mathrm{AL}$ is optimized with respect to the coding capacity at its output, which is restricted by the number of uniglomerular PNs.

The response amplitude observed in individual activated neurons varies across odors, and thus, the amplitude yields additional information above the combinatorial activation pattern. Figure $4 \mathrm{~d}$ estimates the average pairwise Euclidean distance between odor-specific population rate vectors in a time-resolved manner. It shows that the response patterns evoked by different odors build up rapidly and become significantly different after only few tens of milliseconds. The maximum difference in firing rate of average $\sim 30$ spikes per second per neuron is reached after $\sim 200 \mathrm{~ms}$. A recent $\mathrm{Ca}$ imaging study of PNs in the honeybee $\mathrm{AL}$ (Fernandez et al. 2009) reported a somewhat longer time to peak of $375 \mathrm{~ms}$, which is likely due to the slower transients of the $\mathrm{Ca}$ signal. The numbers in the honeybee are in accordance with results obtained from spike train analyses in other species. In the locust odor classification as monitored by extracellular recordings of PN ensembles reached the near-maximum after 100-300 ms (Mazor and Laurent 2005; Stopfer et al. 2003). In Drosophila odor tuning in single PNs was the strongest in the period of 130$230 \mathrm{~ms}$ after stimulus onset (Wilson et al. 2004), and in the moth, a maximal separation of the PN population activity was reached 100 $300 \mathrm{~ms}$ after response onset (Bombyx mori, Namiki and Kanzaki 2008; Namiki et al. 2009; Manduca sexta, Daly et al. 2004; Staudacher et al. 2009).

Principal component analysis of the neural ensemble rate allows visualizing the population response to an odor stimulus as a trajectory in the three-dimensional space spanned by the three major principal components. As shown in Figure 5, the individual odor response trajectories are well separated during stimulation before they return to a common subspace that is occupied by the spontaneous population activity.

\subsection{Latency coding}

There is increasing evidence that a coding scheme of odor-specific response latency patterns might be employed in olfactory systems of vertebrates and invertebrates. The concept of a latency code is simple. A population of neurons is activated in a specific temporal order for one stimulus and in a different order for a different stimulus (Thorpe et al. 2001; Chase and Young 2007). For individual neurons, this scheme implies characteristic odor-specific response latencies. The latency code is reliable, if the pattern of response latencies is stable across stimulus repetitions. It can readily provide a concentration-invariant code for odor identity if this stability of temporal order transfers to different stimulus intensities. Moreover, a latency code is, by definition, fast as the response onsets provide the first datum of the overall ensemble response. A subset of early respond- 


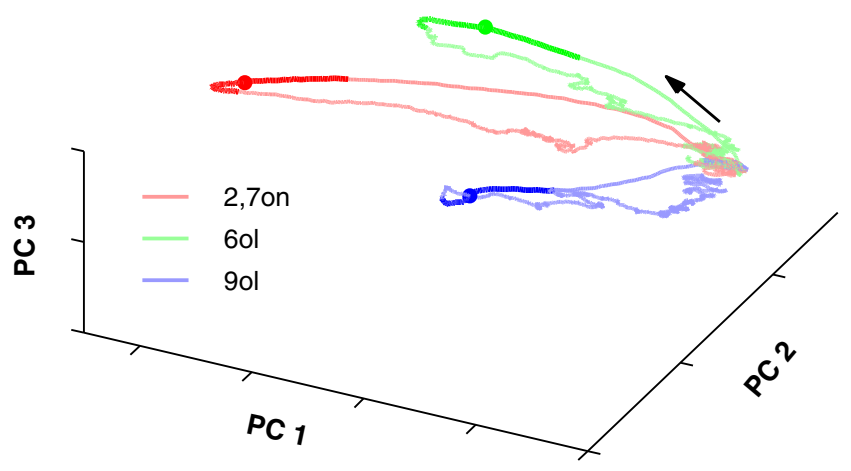

Figure 5. Odor response trajectories visualized in principal component space. A principal component analysis was performed on the firing rate profiles of $N=17$ uniglomerular PNs (Figure 4a-c). The visualized trajectories represent the projection of the 17-dimensional space onto the three major principal components. Each trajectory describes the temporal evolution of the neural population response for one particular odor as indicated. The arrow indicates the direction of time. After stimulus onset the different odor trajectories clearly separate but describe a similar dynamics. Colored circles indicate the point at which the average Euclidean distance between odor pairs reached its maximum; saturated color indicates the episode where the Euclidean distance was above $90 \%$ of its maximum (cf. Figure 4d). The firing rates were estimated using the kernel $\alpha(t)=t \times \exp (-t / \tau)$ with $\tau=50 \mathrm{~ms}$.

ing neurons will provide initially incomplete stimulus information, available much sooner than the fully evolved neural response pattern.

In vertebrates, Spors et al. (2006) first demonstrated by means of voltage sensitive dye imaging in mice that odor-specific response latencies exist already at the level of OSN input to the olfactory bulb. Their results suggest that odor-specific response latencies evolve early on in the periphery, e.g., due to a receptor type specific activation kinetics. More recently, Junek et al. (2010) performed Ca imaging from individual mitral cells in a nose-brain preparation of the frog and demonstrated that the pattern of response latencies carries more information about the odor identity than the respective pattern of response amplitudes.

In insects, visual inspection of single PN spike responses to different odor stimuli give the impression that different odors yield different response onset times in the same neuron (e.g., Perez-Orive et al. 2002; Müller et al. 2002; Wilson et al. 2004). Likewise, the spike responses of a PN population to the same odor stimulus can show neuron-specific response latencies (e.g., Wilson et al. 2004; Namiki and
Kanzaki 2008) suggesting that the population of PNs may indeed employ a latency code. In the honeybee, two studies (Müller et al. 2002; Krofczik et al. 2008) provided statistical evidence for a latency code in uniglomerular PNs by showing that the variation of response latencies across trials was significantly smaller than the variation across odors in single PNs. Notably, the results in Krofczik et al. (2008) on inter-trial and inter-odor latency variance quantitatively matched those obtained in mice (Spors et al. 2006). This observation in the honeybee relies on relatively small data sets, and thus, further investigation is needed, preferably on the basis of simultaneous extracellular recordings from many PNs in the lateral and median tracts (see Section 6). Interestingly, odor-specific latencies in the honeybee are also observable at the level of MB output neurons (Strube-Bloss et al. 2011). Meanwhile, the possibility of an olfactory latency code has also been implicated for other insects, in particular for the moth (Belmabrouk et al. 2011; Kuebler et al. 2011).

As in rodents (Spors et al. 2006), temporal patterning of response onsets in insects is already observed at the level of ORNs (e.g., 
Nagel and Wilson 2010; Raman et al. 2010; Jarriault et al. 2010), and thus, latency patterns at the level of second-order neurons might at least partly be inherited from OSNs. Any statistical result demonstrating that the temporal response pattern across olfactory neurons correlates with odor identity does, of course, not answer the question whether a latency code is actually used by downstream networks or whether this information remains a nuisance to the system. Recently, Smear et al. (2011) provided first evidence that differences in temporal latencies do matter behaviorally. They could show that mice can learn to discriminate fine temporal differences in the experimentally controlled activation of OSNs.

\subsection{Post-stimulus odor representation in the $P N$ population}

In classical Pavlovian conditioning experiments a conditioned stimulus (CS) is paired with an unconditioned stimulus (US) where both stimuli partially overlap in time (delay conditioning). In the trace conditioning paradigm, the US occurs only after the CS has terminated, i.e., there exists a temporal gap between CS and US presentation. Honeybees are able to form odor-reward associations in trace conditioning experiments, albeit with reduced group-averaged learning rates as compared to the outcome in delay conditioning experiments. The group performance during the retention test decreases continuously with the length of the CS-US gap. A significant learning effect could be observed for a maximum gap of $\sim 5-7 \mathrm{~s}$ as measured from the CS onset to the US onset, independent of the CS duration (Menzel and Bitterman 1983; Szyszka et al. 2011). Olfactory trace conditioning has also been observed in other insect species, e.g., in moth (Ito et al. 2008) and fruit fly (Galili et al. 2011).

These observations at the behavioral level pose the question of the underlying mechanisms that facilitate learning in the trace conditioning paradigm (Ito et al. 2008; Szyszka et al. 2011). Within the conceptual framework of Hebbian plasticity (Hebb 1949) for associative learning, synaptic plasticity is triggered if pre- and postsynaptic neurons are active at the same time. For plasticity to take place in the trace conditioning paradigm, this hypothesis implies that the sensory network upstream to the site of Hebbian-type synapses retains stimulus-related activity throughout the CS-US gap, thus representing a long-lasting odor trace.

In their recent study, Szyszka et al. (2011) searched for a neural correlate of this odor trace in the honeybee $\mathrm{AL}$ by means of $\mathrm{Ca}$ imaging from uniglomerular 1-PNs during trace conditioning. They found stimulus-specific activity during the CS-US gap, but a detailed correlation analysis showed that the post-stimulus glomerular response pattern was uncorrelated with the initial CS response pattern. Consequently, perceived odor quality could be predicted from the imaged data during CS presentation, but not after. The authors concluded that the population of uniglomerular 1-PNs does not encode the CS trace that could subserve Hebbian-type plasticity.

Inspired by this recent work, we reanalyzed the data set presented in Krofczik et al. (2008) with respect to the existence of a post-stimulus odor trace in the population activity of uniglomerular PNs, which in this case mainly consists of m-PNs (13 out of 17; Figure 4, right panels). As shown in Figure 4d, the Euclidean distance between population responses to different odors remains at an elevated level after stimulus offset for several seconds. The initial neuronal response pattern (measured at the time of the maximal Euclidean distance) is well correlated with the activity pattern at later stimulus times but this correlation decreases rapidly with stimulus offset assuming small values that are only slightly higher than those for cross-odor correlation (Figure $4 \mathrm{~d}$, right panel). Close inspection of individual PN responses during and after stimulation reveals that only a small subset of the initially excited neurons maintained an elevated firing rate, while the activity of most neurons dropped to baseline or even below. This finding is in agreement with the results in Szyszka et al. (2008). Thus, neither the Ca-activation pattern 
across glomeruli innervated by 1-PNs nor the spiking activity in the population of m-PNs indicated the existence of a reliable representation of the initial stimulus pattern at poststimulus times. However, the analysis presented in Figure 4 was based on a pseudo-population of PNs, each recorded in a different animal. Thus, we cannot exclude that a stable odor representation at the level of PNs existed in some individual bees but not in others (Pamir et al. 2011). In moth, Ito et al. (2008) could show that also Kenyon cell (KC) responses do not represent a persistent odor code.

Taken together, these results provide evidence for the lack of a persistent odor trace carried by neural response activity that could subserve Hebbian plasticity downstream of uniglomerular PNs. However, more intricate neural representations of an odor trace that are not easily observable in a stimulus-triggered analysis might involve dynamic attractors within the AL network representing a more subtle post-stimulus odor trace carried by the network activity (Galán et al. 2006).

\section{RECENT MODEL APPROACHES TO ODOR CODING IN THE HONEYBEE OLFACTORY PATHWAY}

Here, we review recent model approaches concerned with the differential encoding in the dual olfactory pathway in the honeybee and the temporal response evolution across successive layers of the olfactory pathway.

\subsection{A functional role for the dual olfactory pathway}

The existence of a dual odor coding pathway in most Hymenoptera raises the question of its evolutionary benefit (Rössler and Zube 2011). The available experimental evidence indicates that there is no apparent segregation with respect to the encoded odor spectra (Müller et al. 2002; Krofczik et al. 2008; Yamagata et al. 2009; Brandstätter and Kleineidam 2011). The work by Peele et al. (2006) showed that honeybees could not recognize and adequately respond to a learned odor if the 1-APT fiber tract was disrupted, indicating that the two tracts process and transmit complementary stimulus information. The currently prevailing hypothesis is that the dual pathway is functionally optimized for the parallel processing of complex olfactory information, possibly reflecting specific ecological demands in Hymenoptera (Rössler and Zube 2011).

In their recent modeling approach, Schmuker et al. (2011) formulated the hypothesis that mAPT and 1-APT odor processing is differentially tuned to achieve the parallel encoding of odor intensity and odor identity, respectively. This was motivated by three physiological differences observed in PNs of the two tracts (cf. Section 3), namely (1) m-PNs display a broader odor tuning than 1-PNs (but see Müller et al. 2002), (2) m-PNs exhibit a stronger concentration dependence while 1-PNs show a comparably weak concentration dependence (Yamagata et al. 2009), and (3) m-PNs predominantly show hypoadditive mixture encoding where the mixture response is at level with the response to the dominant mixture component, while 1-PNs can show suppressive mixture coding. The authors formalized this hypothesis in a computational model of local inter-glomerular processing in the honeybee AL. In their model, the inhibitory interneuron network accounts for two computational principles, lateral inhibition and gain control, which are differently pronounced in the 1-APT and the m-APT pathways.

Lateral inhibition represents a general and commonly assumed computational principle in neural networks. Experimental results indicate that lateral inhibition within the $\mathrm{AL}$ increases the separability of glomerular response patterns in the fly (Wilson and Laurent 2005; Silbering and Galizia 2007) and enhances odor discriminability in honeybees (Stopfer et al. 1997). Previous model approaches demonstrated that lateral inhibition can indeed increase the separability of glomerular output patterns in the antennal lobe (Linster and Smith 1997; Linster et al. 2005; Beyeler et al. 2010; Häusler et al. 
2011) and the olfactory bulb (Urban 2002; Wick et al. 2010). A detailed model study by Linster et al. (2005) assumed a functional organization of the inhibitory network of heterogeneous LNs in the honeybee AL. Lateral inhibition between any pair of glomeruli was modeled in proportion to the linear correlation of their odor-response profiles as determined on the basis of $\mathrm{Ca}$ imaging experiments of glomerular input. This approach could best explain the transformation of glomerular input patterns (OSN input) into glomerular output patterns (PN output) in the 1APT, outperforming models with a random or morphologically restricted connectivity scheme.

Schmuker et al. (2011) assumed in their model that lateral inhibition and gain control are differently tuned in the m-APT and 1-APT pathways. Using a fix underlying network structure, they adopted the principle of nonuniform correlation-based lateral inhibition mediated by heterogeneous LNs (Linster et al. 2005). Gain control was implemented globally such that the overall PN activity scales global feedback inhibition. Strong gain control resulted
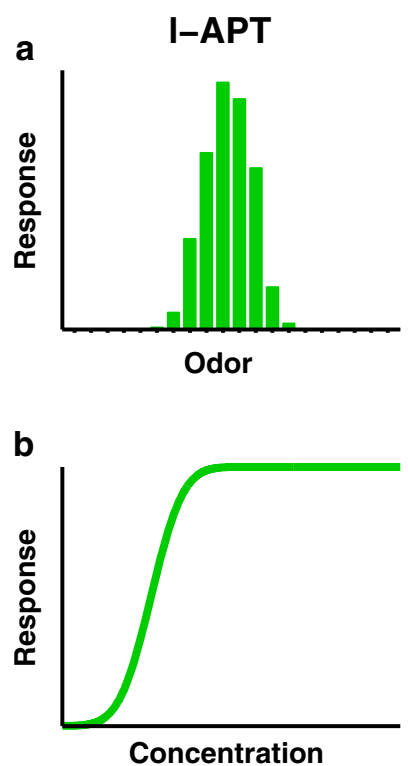

in an almost concentration-invariant output activity. If this was combined with strong lateral inhibition, narrow odor response spectra and relative concentration invariance were observed in individual glomerular output, representing the properties of 1-PNs as schematized in Figure 6 (left). On the contrary, if lateral inhibition and gain control were weak, the response spectra of individual glomeruli were broad and showed pronounced concentration dependence, resembling the properties of $\mathrm{m}-\mathrm{PN}$ output (Figure 6, right). In effect, the spatial activation pattern of 1-APT glomeruli was more distinct for different odors. Finally, presentation of odor mixtures resulted in a synthetic mixture representation in the 1-APT, and the glomerular response pattern of the mixture was distinct from those of the individual components. In mAPT glomeruli, the mixture representation was elemental, i.e., the response patterns were additive or hypoadditive, dominated by the response to the most effective element of the mixture. This model result on mixture coding is in line with previous experimental observations
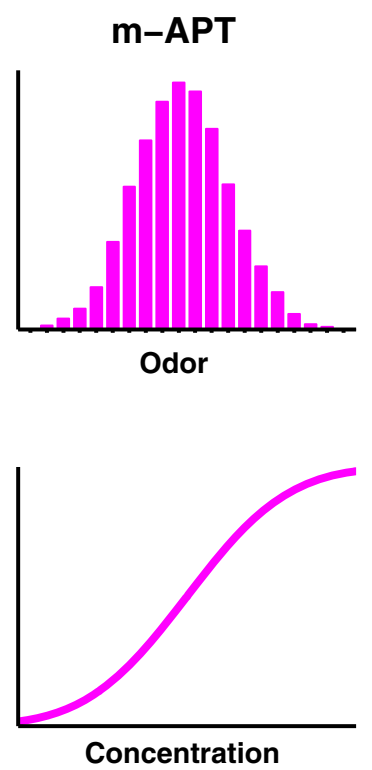

Figure 6. A dual role for the dual odor pathway. Left odor tuning in PNs of the 1-APT is narrower and concentration dependence is weak due to strong lateral inhibition and gain control. Right PNs of the m-APT display a broad odor spectrum (top) and pronounced concentration dependence. 
(Yamagata et al. 2009; Krofczik et al. 2008) and reflects the suggested role for dual pathway mixture coding in Deisig et al. (2010).

In summary, the model assumed the same network substrate for the two AL hemilobes associated with the two pathways. Differential encoding in the m-APT and the l-APT were brought about by parametric variation of two key elements, lateral inhibition and gain control. The authors suggest that these functional specifications of the 1-APT and m-APT could subserve the behavioral task of foraging where independent evidence about odor identity and intensity might be of advantage.

For the local interneuron network this model predicts (a) a functional segregation (lateral inhibition and gain control) and (b) a partial separation between the two hemilobes. For the fruit fly and based on optophysiological observations, Silbering and Galizia (2007) suggested a similar functional division into a global inhibitory network exerting gain control and a glomerulus specific network responsible for lateral inhibition as evident during mixture suppression. In the honeybee, such a functional division might be supported by the two major morphological classes of homogeneous and heterogeneous LNs. The former exhibit a global branching pattern innervating a large number of glomeruli suited for global gain control. The latter show a dense innervation in one glomerulus and a sparse innervation of several other glomeruli suited for glomerular specific lateral inhibition (Fonta et al. 1993).

\subsection{Phasic-tonic response dynamics with spike frequency adaptation}

Olfactory information is transmitted across successive stages of the olfactory pathway. At each stage, a different temporal response characteristic is observed that becomes progressively phasic from OSNs to PNs to KCs.

At the peripheral level of olfactory encoding, OSNs exhibit a dominant phasic-tonic response component in reaction to a constant odor stimulus. This has been observed in different insect species (e.g., de Bruyne et al. 1999;
Hallem and Carlson 2006; Bhandawat et al. 2007; Raman et al. 2010; Jarriault et al. 2010; Nagel and Wilson 2010) and in vertebrates (e.g., Friedrich and Laurent 2001). In a detailed experimental study, Nagel and Wilson (2010) decomposed single OSN responses in the fruit fly into a two-step process of transduction and spike generation. The former could be adequately described by a linear low-pass filter, the latter by a linear filter which effectively implements a time derivative of the transduction signal.

One plausible biophysical explanation for a phasic-tonic neuronal response characteristic is a neuron-intrinsic mechanism of self-inhibition. Spike frequency adaptation (SFA) is a prominent phenomenon of self-inhibition and ubiquitous in invertebrate and vertebrate neurons (Benda and Herz 2003; Benda and Hennig 2008; Farkhooi et al. 2009a; Benda et al. 2010). Different currents have been described that can mediate SFA, and a variety of computational single neuron models for SFA have been devised at different levels of abstraction (for review, see Farkhooi et al. 2009a; Benda et al. 2010). In a recent study of olfactory response dynamics, Farkhooi et al. (2009b, 2010) devised the neuron model described by Müller et al. (2007), which resembles a conductance based integrate-and-fire neuron that incorporates an additional slow SFA conductance. As depicted in Figure $7 \mathrm{a}$, each single action potential causes a step-wise increment of the hyperpolarizing SFA conductance (black curve) by a fix quantum $q$ (red steps), which decays exponentially with time constant $\tau$. A rapid succession of action potentials in response to the stimulus onset will accumulate the SFA conductance and, thus, self-inhibit the neuron, progressively reducing the rate of action potential generation until it reaches the steady state. Figure $7 \mathrm{~b}$ shows the neuron's response behavior when stimulated for $2 \mathrm{~s}$ with discrete excitatory events that followed a Poisson process, reminiscent of stochastic transduction events in OSN dendrites (Nagel and Wilson 2010). The neuron responds to stimulus onset with an initial phasic component before it approaches the adaptation rate. At 
a
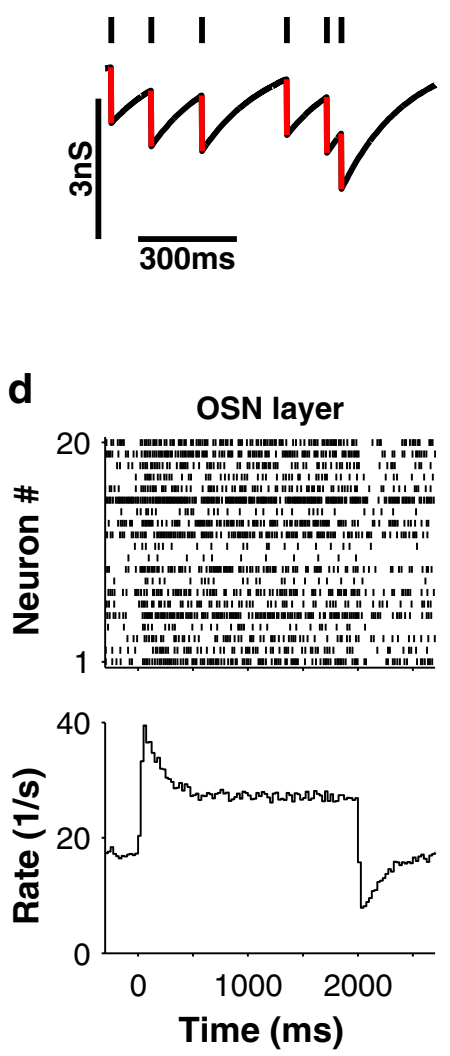

b

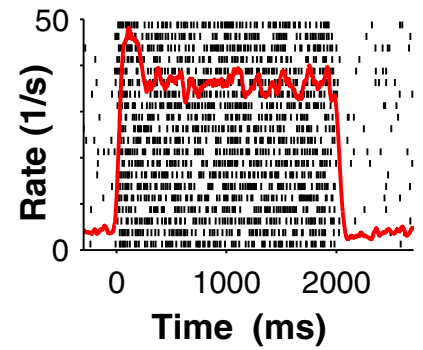

e
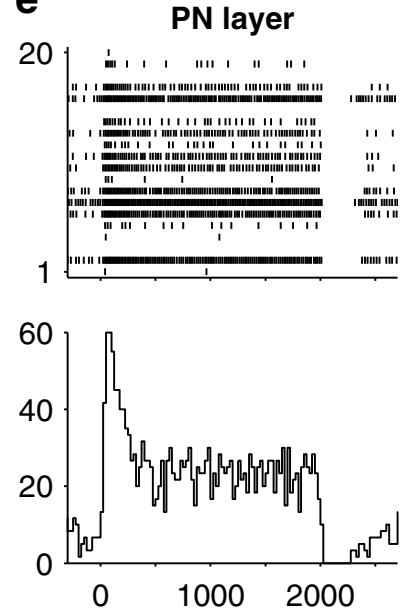

C
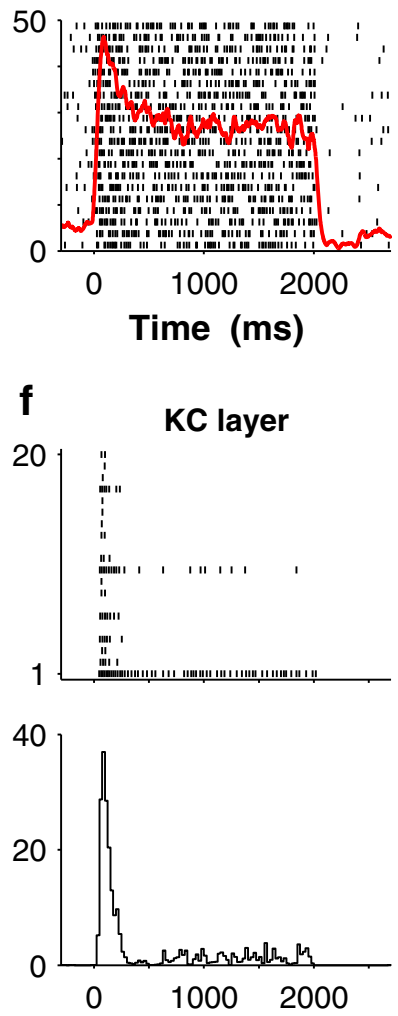

Figure 7. Dynamic response model for the olfactory pathway. a Conductance-based model of SFA. Each action potential (top) induces a small step increase $q$ (red) of the outward SFA conductance (black curve), which decays exponentially with time constant $\tau$. b Spike trains (black ticks) during 20 repeated simulations and estimated firing rate (red curve). For SFA parameters $q=1.2 \mathrm{nS}$ and $\tau=200 \mathrm{~ms}$ this results in a phasic-tonic rate profile. c Increasing the effect of the SFA conductance ( $q=1.5 \mathrm{nS}$ and $\tau=400 \mathrm{~ms})$ yields a more pronounced adaptation of the tonic response rate. The trial-to-trial variability of the spike response is reduced with increasing strength of SFA yielding a Fano factor of 0.28 in $\mathbf{b}$ and 0.14 in $\mathbf{c}$. $\mathbf{d}-\mathbf{f}$ Dynamic population response across successive layers of the olfactory network. Top panels indicate simultaneous spike train responses of 20 single neurons in each layer; bottom panels show the estimated population rate averaged across all neurons in the respective layer. OSNs (d) and PNs (e) show a phasic-tonic population response due to SFA. $\mathbf{f}$ Individual Kenyon cell responses are temporally sparse due to a strong SFA conductance, which results in a sharp transient response in the population rate. d-f Reproduced from simulated data in Farkhooi (2011).

stimulus offset, the SFA conductance causes a short phase of suppressed spiking matching experimental findings in OSNs (e.g., Nagel and Wilson 2010). In effect, the SFA mechanism approximates a temporal differentiation (Lundstrom et al. 2008; Tripp and Eliasmith 2010) and may thus account for the phenomenological observation of a differentiating filter that could well describe the transfer function of the spike encoding mechanism in OSNs of the fruit fly (Nagel and Wilson 2010).

The response behavior of PNs, the secondary olfactory neurons in the insect, exhibit a similar dominant phasic-tonic response component, e.g., in the fruit fly (Bhandawat et al. 2007; Wilson et al. 2004; Olsen et al. 2007), in the silkmoth 
(Namiki and Kanzaki 2008), and in the locust (Stopfer et al. 2003; Mazor and Laurent 2005). In single PNs of the honeybee, this component dominates during the early phase of a stimulus (Figure 2a, Krofczik et al. 2008). This response profile becomes more evident in the population response (Figure $2 \mathrm{~b}, \mathrm{c}$ ) and causes a phasic-tonic evolution of the population code (Euclidean distance in Figure 4e). At the cellular level, in vitro recordings from dissociated and cultured neurons from pupae of the honeybee (Grünewald 1993) and the moth (M. sexta, Mercer and Hildebrand 2002) have described calciumdependent $\mathrm{K}+$ currents in PNs, which suggests a possible SFA mechanism (Sivan and Kopell 2006; Belmabrouk et al. 2011). Direct comparison of OSN and PN responses showed that the phasic component is more pronounced in PNs than in OSNs (e.g., Wilson et al. 2004). In the model neuron of Müller et al. (2007), this can be achieved by increasing the SFA parameters $q$ and $\tau$ which increases the effect of self-inhibition resulting in a stronger phasic response component and a lower adaptation rate as demonstrated in Figure 7c.

Beyond the initial phasic-tonic response component, PN spiking responses exhibit slow patterning during the later phases of a continued stimulation (Figure 2a, Sun et al. 1993; Abel et al. 2001; Müller et al. 2002). This might be due to alternating phases of inhibition and excitation during continued stimulation. However, the model results of Sivan and Kopell (2006) suggest that a SFA mechanism in PNs might suffice to explain slow patterning. Similar, a recent model study by Belmabrouk et al. (2011) showed that self-inhibiting currents are required to explain experimentally observed multi-phasic responses patterns in PNs.

An important secondary effect of SFA is that it decreases response variability and hence supports reliable signal transmission (Farkhooi et al. 2011; Chacron et al. 2004). The trial-totrial variability of spike train responses is commonly quantified by the Fano factor (Nawrot 2010). It measures the ratio of the cross-trial variance and mean of the trial-to-trial spike count. In the example of Figure 7, the
Fano factor reduces with increasing strength of the adaptation conductance from 0.28 in Figure $7 \mathrm{a}$ to 0.13 in Figure $7 \mathrm{~b}$, and hence, signal reliability is increased.

Alternative self-inhibiting mechanism can imply phasic-tonic responses, e.g., selfinhibition at the synapse level through shortterm depression. This was described at synapses between OSNs and PNs in the fruit fly (Kazama and Wilson 2008; Kazama and Wilson 2009) and can produce a phasic-tonic response in PNs to a constant stimulus (Nawrot et al. 2010).

\subsection{Temporally sparse odor representation in $\mathrm{KCs}$}

The third order olfactory neurons, the KCs, employ a spatially and temporally sparse odor code. This has been demonstrated in the locust (Perez-Orive et al. 2002), the honeybee (Szyszka et al. 2005; Szyszka et al. 2008), the moth (Ito et al. 2008), and the fruit fly (Honegger et al. 2011). Spatial sparseness refers to the fact that each $\mathrm{KC}$ displays a narrow odor spectrum (usually referred to as lifetime sparseness) and, in turn, each odor activates only a very small subset of KCs (population sparseness). This can be explained by the anatomical connection scheme where a relatively small number of PNs, in the honeybee 2920 uniglomerular PNs of both tracts, make divergentconvergent connections with a much larger (in the honeybee approximately 100-fold) $\mathrm{KC}$ population and by a lower sensitivity of the KCs (e.g., Jortner et al. 2007). Numbers of PN inputs per $\mathrm{KC}$ estimated from physiological recordings vary from 10 (or $2 \%$ ) for clawed KCs (Szyszka et al. 2005) to 400 (or $~ 50 \%$ ) in locust (Jortner et al. 2007).

Temporal sparseness refers to the fact that $\mathrm{KCs}$ produce only very few spikes in response to stimulus onset resulting in a brief transient population response (Perez-Orive et al. 2002; Broome et al. 2006; Szyszka et al. 2005; Ito et al. 2008; Honegger et al. 2011). In the locust, it has been shown that PN output is locked to strong oscillations carried by the inhibitory interneuron network (Perez-Orive et al. 2002) 
and that feed-forward inhibition via lateral horn interneurons together with $\mathrm{MB}$ intrinsic inhibitory feedback truncates $\mathrm{KC}$ responses (Assisi et al. 2007; Papadopoulou et al. 2011), effectively implementing a mechanism of coincidence detection that might facilitate a temporal coding scheme.

In the honeybee, it was suggested that inhibitory feedback of MB output neurons in the proto-cerebral-tract could mediate temporal sparseness of Kenyon cells (Szyszka et al. 2005). Farkhooi et al. (2009b, 2010) alternatively suggested a multi-layer feed-forward model of the honeybee olfactory pathway that can reproduce population and temporal sparseness in Kenyon cells without assuming inhibitory feed-forward or feedback inhibition. Their neural network model involves three layers consisting of $\sim 1,500 \mathrm{OSNs}, 24 \mathrm{PNs}$, and 100 $\mathrm{KCs}$, respectively. The neuron numbers are in relative proportion to the anatomically estimated numbers (cf. Section 2). The network structure assumes a simplified connectivity scheme where the OSNs make convergent random connections with the PNs. The connectivity from the PNs to the $\mathrm{KCs}$ is divergentconvergent and random where each $\mathrm{KC}$ receives input from $50 \%$ of all PNs. All neurons are modeled by the conductance-based integrated and fire neuron with SFA (Figure 7a, Müller et al. 2007), albeit with increasing strength of SFA from the OSN to the PN to the $\mathrm{KC}$ layer.

The temporal evolution of the network response to a constant odor stimulus is reproduced in Figure $7 d-f$. Each OSN in the first layer was constantly excited by a stochastic train of transduction events modeled by a Poisson process. Each event caused a phasic conductance increase, the strength of which was heterogonous across the OSN population. During stimulus presentation, the Poisson intensity was increased by a fix amount for each neuron. As a result, individual OSNs responded with different amplitude (Figure 7d). The average firing rate in the OSN population in Figure $7 \mathrm{~d}$ reveals the SFA mediated phasictonic adaptation profile and suppression of spike output after stimulus offset. Due to the convergent but random connections from the first to the second layer, only a subset of PNs exhibited a significant excitatory response. Increased SFA conductance in the PN layer resulted in a more pronounced phasic response component in the population histogram (Figure 7e). In the third layer, individual KCs typically respond with only very few spikes (Figure $7 \mathrm{f}$ ). This is due to a considerably strong SFA conductance with long adaptation time constant that leads to rapid response suppression. Experimental support for strong SFA currents in KCs has been provided by Demmer and Kloppenburg (2009) who studied in detail the physiology of KCs of the cockroach. The KC population response in the model resembles experimental observations for the KC population activity (Ito et al. 2008; Szyszka et al. 2005). In the model, each KC produces on average 2.4 spikes during the $2 \mathrm{~s}$ stimulus interval and the first $600 \mathrm{~ms}$ after stimulus onset comprises $\sim 65 \%$ of the total number of response spikes, which quantitatively matches the observation by Ito et al. (2008).

Mechanisms for temporal sparseness involving feed-forward or feedback inhibition as outlined above and the mechanism proposed by Farkhooi and colleagues are not mutually exclusive but may act in concert. If SFA would indeed play a dominant role in regulating temporal sparseness in the honeybee, the experimental prediction would be that systemic blocking of inhibitory transmission would affect response strength and spatial sparseness, both in the $\mathrm{PN}$ and the $\mathrm{KC}$ layer, but not temporal sparseness in the KC layer (Farkhooi 2011).

Importantly, the reduction in single neuron variability due to SFA (cf. Section 5.2) transfers to the population activity (Farkhooi et al. 2011). This is of relevance because each neuron in the downstream network layer receives input from a population of presynaptic neurons of the previous layer. Thus, SFA increases the reliability of both single neuron output and the population synaptic input to the postsynaptic neuron and hence improves the reliability of signal transmission in all layers.

The simple network architecture with successive stages of fast neural adaptation can approx- 
imate the mathematical operation of temporal differentiation such that the $\mathrm{KC}$ output encodes the time derivative of the input (Tripp and Eliasmith 2010). This might suggest that the olfactory system of the insect could be designed to focus on temporal changes and to largely ignore constancy in the olfactory input. The experimental prediction is that, under naturally dynamic input conditions, the activity of single $\mathrm{KCs}$ is not temporally sparse but rather driven by the naturally occurring fluctuations of odor composition and odor concentration.

\section{CONCLUSIONS AND OUTLOOK}

While the basic blueprint of the olfactory system is similar across different insect species and despite the fact that the homology of the insect and the mammalian olfactory system has been stressed in numerous publications, it is increasingly acknowledged that the speciesspecific specialties expressed in anatomical, morphological, and physiological features likely find expression in specialized functions and different coding strategies (Daly et al. 2011; Blumhagen et al. 2011) that are specifically tailored to the different ecological demands (Martin et al. 2011; Galizia and Rössler 2010; Rössler and Zube 2011; Hansson and Stensmyr 2011).

It is now widely accepted that the insect AL does not merely act as a relay station or linear filter of peripheral input. Here, we reviewed the experimental evidence for complex and nonlinear odor processing in the honeybee AL network that reflects the complexity and temporal dynamics of the olfactory input. The specific function of the dual pathway still remains unresolved. With some certainty it can be excluded that it reflects a division in the general odor profile (cf. Section 3.2) as, for example, the division in a general odor and a specialized pheromone system in moths. More intricate differences with respect to the mixture suppression effect, odor specificity, and concentration dependence and subtle differences in single neuron response patterns mostly rely on rather small experimental sample sizes due to the difficulties of stable in vivo intracellular recordings and neuron identification. New methods of extracellular recordings from insect brains have been developed that allow stable recording of multiple single units and they have been successfully applied to record MB output neurons in the honeybee (Okada et al. 2007; Strube-Bloss et al. 2011). Interesting results can be expected from the adaptation of these methods to the simultaneous extracellular PN recordings in both 1-APT and $\mathrm{m}$-APT fiber tracts (Brill et al. 2011).

An improved understanding of stimulus processing under natural conditions requires experimental studies under conditions that approximate natural stimuli. This approach has revolutionized our view on sensory processing in mammalian sensory systems (e.g., Felsen and Dan 2004). The natural olfactory environment of the honeybee is complex and highly dynamic. The classical stimulation protocol used in the laboratory for decades, however, used constant odor stimulation. A number of recent studies have used novel stimulation protocols with time-varying olfactory stimuli, mostly realized through a controlled pattern of rapid valve opening and closing (e.g., Geffen et al. 2009; Nagel and Wilson 2010; Meyer and Galizia (2012)). Here, computational approaches could make major contributions by testing models of neural information processing in virtual olfactory environments, which simulate the turbulent and filamental structure of odor plumes in order to mimic the spatiotemporal structure and dynamics of point-like and wide-field odor sources that insects encounter in their natural environment.

\section{ACKNOWLEDGMENTS}

I thank Sabine Krofczik and Randolf Menzel for providing me with the electrophysiological data that was reproduced in Figures 2, 3, 4, and 5 and Farzad Farkhooi for carrying out the simulations shown in Figure 7. I am grateful to Jürgen Rybak for the 3D visualization of neurons in the Honeybee Standard Brain atlas (Figure 1) and for his helpful comments 
on an earlier version of this manuscript. I thank Randolf Menzel, Anneke Meyer, Paul Szyszka and Michael Schmuker for valuable discussions and Chris Häusler for the language check. We acknowledge generous funding from the German Federal Ministry of Education and Research to the Bernstein Focus Learning and Memory-Insect inspired robots (01GQ0941) and to the Bernstein Center for Computational Neuroscience Berlin (01GQ1001D). Dynamique du traitement sensoriel dans les voies olfactives dédoublées chez l'abeille.

Lobe antennaire / codage raréfié / codage de la latence / codage de la trace / olfaction

\section{Dynamik sensorischer Verarbeitung im dualen olfaktorischen Pfad der Honigbiene}

\section{Antennallobus / spärliche Kodierung / Latenzkodierung / Duftspur / Geruchssinn}

\section{REFERENCES}

Abel, R., Rybak, J., Menzel, R. (2001) Structure and response patterns of olfactory interneurons in the honeybee, Apis mellifera. J. Comp. Neurol. 437, 363383

Abraham, N.M., Spors, H., Carleton, A., Margrie, T.W., Kuner, T., Schaefer, A.T. (2004) Maintaining accuracy at the expense of speed: stimulus similarity defines odor discrimination time in mice. Neuron 44, 865-876

Assisi, C., Stopfer, M., Laurent, G., Bazhenov, M. (2007) Adaptive regulation of sparseness by feedforward inhibition. Nat. Neurosci. 10, 1176-84

Belmabrouk, H., Nowotny, T., Rospars, J.-P., Martinez, D. (2011) Interaction of cellular and network mechanisms for efficient pheromone coding in moths. PNAS 108, 19790-19795

Benda, J., Herz, A.V. (2003) A universal model for spike-frequency adaptation. Neural Comput. 15, 2523-64

Benda, J., Hennig, R.M. (2008) Spike-frequency adaptation generates intensity invariance in a primary auditory interneuron. J. Comput. Neurosci. 24, 113-36

Benda, J., Maler, L., Longtin, A. (2010) Linear versus nonlinear signal transmission in neuron models with adaptation currents or dynamic thresholds. J. Neurophysiol. 104, 2806-20

Beyeler, M., Stefanini, F., Proske, H., Galizia, G., Chicca, E. (2010) Exploring olfactory sensory networks: simulations and hardware emulation. IEEE
Biomedical Circuits and Systems Conference (BioCAS) 2010, 270-273

Bhandawat, V., Olsen, S., Gouwens, N., Schlief, M., Wilson, R. (2007) Sensory processing in the Drosophila antennal lobe increases reliability and separability of ensemble odor representations. Nat. Neurosci. 10, 1474-1482

Blumhagen, F., Zhu, P., Shum, J., Schärer, Y.P.Z., Yaksi, E., Deisseroth, K., Friedrich, R.W. (2011) Neuronal filtering of multiplexed odour representations. Nature 479, 493-500

Brandstätter, A.S., Kleineidam, C. (2011) Distributed representation of social odors indicates parallel processing in the antennal lobe of ants. J. Neurophysiol. 106, 2437-2449

Brill, M. F., Reus, I., Rosenbaum, T., Kleineidam, C. J., Rössler, W. (2011). Simultaneous recordings from multiple projection neurons in the dual olfactory pathway of the honeybee. Proceedings of the 9th Göttingen Meeting of the German Neuroscience Society: T19-31A.

Broome, B.M., Jayaraman, V., Laurent, G. (2006) Encoding and decoding of overlapping odor sequences. Neuron 51(4), 467-82

de Bruyne, M., Clyne, P.J., Carlson, J.R. (1999) Odor coding in a model olfactory organ: the Drosophila maxillary palp. J. Neurosci. 19, 4520-4532

Chacron, M.J., Lindner, B., Longtin, A. (2004) Noise shaping by interval correlations increases information transfer. PRL. doi:10.1103/PhysRevLett.92.080601

Chandra, S., Smith, B.H. (1998) An analysis of synthetic processing of odor mixtures in the honeybee. J. Exp. Biol. 201, 3113-3121

Chase, S.M., Young, E.D. (2007) First-spike latency information in single neurons increases when referenced to population onset. PNAS 104, 5175-80

Daly, K.C., Wright, G.A., Smith, B.H. (2004) Molecular features of odorants systematically influence slow temporal responses across clusters of coordinated antennal lobe units in the moth Manduca sexta. J. Neurophysiol. 92, 236-54

Daly, K.C., Galán, R.F., Peters, O.J., Staudacher, E.M. (2011) Detailed characterization of local field potential oscillations and their relationship to spike timing in the antennal lobe of the moth Manduca sexta. Front. Neuroeng. 4, 12. doi:10.3389/ fneng.2011.00012

Davison, I., Katz, L. (2007) Sparse and selective odor coding by mitral/tufted neurons in the main olfactory bulb. J. Neurosci. 27, 2091-2101

Deisig, N., Lachnit, H., Giurfa, M., Hellstern, F. (2001) Configural olfactory learning in honeybees: negative and positive patterning discrimination. Learn. Mem. 8(2), 70-78

Deisig, N., Lachnit, H., Giurfa, M. (2002) The effect of similarity between elemental stimuli and compounds in olfactory patterning discriminations. Learn. Mem. 9(3), 112-121 
Deisig, N., Lachnit, H., Sandoz, J.C., Lober, K., Giurfa, M. (2003) A modified version of the unique cue theory accounts for olfactory compound processing in honeybees. Learn. Mem. 10(3), 199-208

Deisig, N., Giurfa, M., Lachnit, H., Sandoz, J. (2006) Neural representation of olfactory mixtures in the honeybee antennal lobe. Eur. J. Neurosci. 24, 11611174

Deisig N, Giurfa M, Sandoz JC (2010) Antennal lobe processing increases separability of odor mixture representations in the honeybee. J. Neurophysiol. 103:2185-2194

Demmer, H., Kloppenburg, P. (2009) Intrinsic membrane properties and inhibitory synaptic input of kenyon cells as mechanisms for sparse coding? J Neurophysiol. 102(3), 1538-50

Esslen, J., Kaissling, K. (1976) Zahl und Verteilung antennaler Sensillen bei der Honigbiene Apis mellifera L. Zoomorphologie 83, 227-251

Farkhooi, F., Strube, M., Nawrot, M.P. (2009a) Serial correlation in neural spike trains: experimental evidence, stochastic modelling, and single neuron variability. Phys. Rev. E 79, 021905

Farkhooi, F., Müller, E., Nawrot, M.P. (2009b) Sequential sparsing by successive adapting neural populations. BMC Neurosci. 10(I), O10. doi:10.1186/14712202-10-S1-O10

Farkhooi, F., Müller, E., Nawrot, M.P. (2011) Adaptation reduces variability of the neuronal population code. Phys. Rev. E 83, 050905

Farkhooi F, Muller E, Nawrot MP (2010) Sequential sparsening by successive adaptation in neural populations. arXiv: $1007.2345 \mathrm{v} 1$

Farkhooi F (2011) Emergent properties of spikefrequency adaptation in neuronal systems: nonrenewal statistics, variability reduction and sparsening. Ph.D. thesis, Freie Universität Berlin. http:// www.diss.fu-berlin.de/diss/receive/FUDISS thesis_000000025213

Felsen, G., Dan, Y. (2004) A natural approach to studying vision. Nat. Neurosci. 8, 1643-1646

Fernandez, P.C., Locatelli, F.F., Person-Rennell, N., Deleo, G., Smith, B.H. (2009) Associative conditioning tunes transient dynamics of early olfactory processing. J. Neurosci. 29(33), 10191-202

Friedrich, R.W., Laurent, G. (2001) Dynamic optimization of odor representations by slow temporal patterning of mitral cell activity. Science 291, 889-94

Fujiwara, T., Kazawa, T., Haupt, S.S., Kanzaki, R. (2009) $\mathrm{Ca}^{2+}$ imaging of identifiable neurons labeled by electroporation in insect brains. Chem. Senses 20, 1061-1065

Fonta, C., Sun, X., Masson, C. (1993) Morphology and spatial distribution of bee antennal lobe interneurones responsive to odours. Chem. Senses 18, 101-119

Galán, R.F., Weidert, M., Menzel, R., Herz, A.V., Galizia, C.G. (2006) Sensory memory for odors is encoded in spontaneous correlated activity between olfactory glomeruli. Neural Comput. 18, 10-25

Galili, D.S., Lüdke, A., Galizia, C.G., Szyszka, P., Tanimoto, H. (2011) Olfactory trace conditioning in Drosophila. J. Neurosci. 31, 7240-7248

Galizia, C., Kimmerle, B. (2004) Physiological and morphological characterization of honeybee olfactory neurons combining electrophysiology, calcium imaging and confocal microscopy. J. Comp. Physiol. A 190, 21-38

Galizia, C.G., Sachse, S., Rappert, A., Menzel, R. (1999) The glomerular code for odor representation is species specific in the honeybee Apis mellifera. Nat. Neurosci. 2, 473-8

Galizia, C., Menzel, R. (2000) Odour perception in honeybees: coding information in glomerular patterns. Curr. Opin. Neurobiol. 10, 504-510

Galizia, C.G., Rössler, W. (2010) Parallel olfactory systems in insects: anatomy and function. Annu. Rev. Entomol. 55, 399-420

Ganeshina, O., Menzel, R. (2001) GABAimmunoreactive neurons in the mushroom bodies of the honeybee: an electron microscopic study. J. Comp. Neurol. 437(3), 335-349

Geffen, M.N., Broome, B.M., Laurent, G., Meister, M. (2009) Neural encoding of rapidly fluctuating odors. Neuron 61, 570-86

Grünewald, B. (1993) Differential expression of voltagesensitive $\mathrm{K}+$ and $\mathrm{Ca} 2+$ currents in neurons of the honeybee olfactory pathway. J. Exp. Biol. 206, 117129

Guerrieri, F., Schubert, M., Sandoz, J., Giurfa, M. (2005) Perceptual and neural olfactory similarity in honeybees. PLoS Biol. 3, e60

Haase, A., Rigosi, E., Trona, F., Anfora, G., Vallortigara, G., Antolini, R., Vinegoni, C. (2010) In-vivo twophoton imaging of the honey bee antennal lobe. Biomed. Opt. Express 2, 131-8

Hallem, E.A., Carlson, J.R. (2006) Coding of odors by a receptor repertoire. Cell 125, 143-60

Hansson, B.S., Stensmyr, M.C. (2011) Evolution of insect olfaction. Neuron 72, 698-711

Häusler C, Nawrot MP, Schmuker M (2011) A spiking neuron classifier network with a deep architecture inspired by the olfactory system of the honeybee. Proceedings of the 5th International IEEE EMBS Conference on Neural Engineering, Cancun, Mexico, April 27-May 1, 2011: 198-202

Hebb, D.O. (1949) The organisation of behavior. Wiley, New York

Holt, G.R., Softky, W.R., Koch, C., Douglas, R.J. (1996) Comparison of discharge variability in vitro and in vivo in cat visual cortex neurons. J. Neurophysiol. 75, 1806-1814

Honegger, K.S., Campbell, R.A.A., Turner, G.C. (2011) Cellular-resolution population imaging reveals ro- 
bust sparse coding in the Drosophila mushroom body. J. Neurosci. 31, 11772-11785

Ito, I., Ong, R.C., Raman, B., Stopfer, M. (2008) Sparse odor representation and olfactory learning. Nat. Neurosci. 11(10), 1177-84

Jarriault, D., Gadenne, C., Lucas, P., Rospars, J.-P., Anton, S. (2010) Transformation of the sex pheromone signal in the noctuid moth agrotis ipsilon: from peripheral input to antennal lobe output. Chem. Senses 35, 705-715

Joerges, J., Küttner, A., Galizia, C.G., Menzel, R. (1997) Representation of odours and odour mixtures visualized in the honeybee brain. Nature 387, 285-288

Jortner, R.A., Farivar, S.S., Laurent, G. (2007) A simple connectivity scheme for sparse coding in an olfactory system. J. Neurosci. 27, 1659-69

Junek, S., Kludt, E., Wolf, F., Schild, D. (2010) Olfactory coding with patterns of response latencies. Neuron 67, 872-884

Kazama, H., Wilson, R.I. (2008) Homeostatic matching and nonlinear amplification at identified central synapses. Neuron 58(3), 401-13

Kazama, H., Wilson, R.I. (2009) Origins of correlated activity in an olfactory circuit. Nat. Neurosci. 12, 1136-44

Kirschner, S., Kleineidam, C., Zube, C., Rybak, J., Grünewald, B., Roessler, W. (2006) Dual olfactory pathway in the honeybee, Apis mellifera. J. Comp. Neurol. 499, 933-952

Krofczik, S., Menzel, R., Nawrot, M.P. (2008) Rapid odor processing in the honeybee antennal lobe network. Front. Comput. Neurosci. 2, 9

Kuebler, L.S., Olsson, S.B., Weniger, R., Hansson, B.S. (2011) Neuronal processing of complex mixtures establishes a unique odor representation in the moth antennal lobe. Front. Neural Circuits 5, 7

Laurent, G., Davidowitz, H. (1994) Encoding of olfactory information with oscillating neural assemblies. Science 265, 1872-5

Linster, C., Smith, B.H. (1997) A computational model of the response of honey bee antennal lobe circuitry to odor mixtures: overshadowing, blocking and unblocking can arise from lateral inhibition. Behav. Brain Res. 87, 1-14

Linster, C., Sachse, S., Galizia, C.G. (2005) Computational modeling suggests that response properties rather than spatial position determine connectivity between olfactory glomeruli. J. Neurophys. 93, 3410-3417

Lundstrom, B.N., Higgs, M.H., Spain, W.J., Fairhall, A. L. (2008) Fractional differentiation by neocortical pyramidal neurons. Nat. Neurosci. 11, 1335-1342

Martin, J.P., Beyerlein, A., Dacks, A.M., Reisenman, C. E., Riffell, J.A., Lei, H., Hildebrand, J.G. (2011) The neurobiology of insect olfaction: sensory processing in a comparative context. Prog. Neurobiol. 95, 427447
Mazor, O., Laurent, G. (2005) Transient dynamics versus fixed points in odor representations by locust antennal lobe projection neurons. Neuron 48, 661673

Menzel, R., Bitterman, M.E. (1983) Learning by honey bees in an unnatural situation. In: Huber, F., Mark1, H. (eds.) Neuroethology and Behavioral Physiology, pp. 206-215. Springer, Berlin

Mercer, A.R., Hildebrand, J.G. (2002) Developmental changes in the electrophysiological properties and response characteristics of Manduca antennal-lobe neurons. J. Neurophysiol. 87, 2650-2663

Meyer A (2011) Characterisation of local interneurons in the antennal lobe of the honeybee. Dissertation, University of Konstanz, Germany. http://nbnresolving.de/urn:nbn:de:bsz:352-162535

Meyer, A., Galizia, C.G. (2012) Elemental and configural olfactory-coding by antennal lobe neurons of the honey bee (Apis mellifera). J. Comp. Physiol. A 198(2), 159-171

Meyer, A., Galizia, C., Nawrot, M.P. (2011) A spiking point of view-is it possible to predict a neurons morphology from its electrophysiological activity? Front. Comput. Neurosci.. doi:10.3389/conf. fncom.2011.53.0015. Conference Abstract

Müller, E., Buesing, L., Schemmel, J., Meier, K. (2007) Spike-frequency adapting neural ensembles: beyond mean adaptation and renewal theories. Neural Comput. 19(11), 2958-3010

Müller, D., Abel, R., Brandt, R., Zoeckler, M., Menzel, R. (2002) Differential parallel processing of olfactory information in the honeybee, Apis mellifera $L$. J. Comp. Physiol. A 188, 359-370

Nagel, K.I., Wilson, R.I. (2010) Biophysical mechanisms underlying olfactory receptor neuron dynamics. Nat. Neurosci. 14, 208-218

Namiki, S., Kanzaki, R. (2008) Reconstructing the population activity of olfactory output neurons that innervate identifiable processing units. Front. Neural Circuits 2, 1

Namiki, S., Haupt, S.S., Kazawa, T., Takashima, A., Ikeno, H., Kanzaki, R. (2009) Reconstruction of virtual neural circuits in an insect brain. Front. Neurosci. 3(2), 206-13

Nawrot, M.P. (2010) Analysis and interpretation of interval and count variability in neural spike trains. In: Grün, S., Rotter, S. (eds.) Analysis of Parallel Spike Trains. Springer, New York

Nawrot MP, Krofczik S, Farkhooi F, Menzel R (2010) Fast dynamics of odor rate coding in the insect antennal lobe. arXiv:1101.0271v1

Okada, R., Rybak, J., Manz, G., Menzel, R. (2007) Learning-related plasticity in PE1 and other mushroom body-extrinsic neurons in the honeybee brain. J. Neurosci. 27, 11736-47

Olsen, S.R., Bhandawat, V., Wilson, R.I. (2007) Excitatory interactions between olfactory processing 
channels in the Drosophila antennal lobe. Neuron 54, 89-103

Pamir, E., Chakroborty, N.K., Stollhoff, N., Gehring, K. B., Antemann, V., Morgenstern, L., Felsenberg, J., Eisenhardt, D., Menzel, R., Nawrot, M.P. (2011) Average group behavior does not represent individual behavior in classical conditioning of the honeybee. Learn. Mem. 18, 733-741

Papadopoulou, M., Cassenaer, S., Nowotny, T., Laurent, G. (2011) Normalization for sparse encoding of odors by a wide-field interneuron. Science 332, 721-5

Peele P, Ditzen M, Menzel R, Galizia CG (2006) Appetitive odor learning does not change olfactory coding in a subpopulation of honeybee antennal lobe neurons. J Comp Physiol A 192: 1083-1103. doi:10.1007/s00359-006-0152-3

Pelz, C., Gerber, B., Menzel, R. (1997) Odorant intensity as a determinant for olfactory conditioning in honeybees: roles in discrimination, overshadowing and memory consolidation. J. Exp. Biol. 200, 837847

Perez-Orive, J., Mazor, O., Turner, G., Cassenaer, S., Wilson, R., Laurent, G. (2002) Oscillations and sparsening of odor representations in the mushroom body. Science 297, 359-365

Ponce-Alvarez, A., Kilavik, B.E., Riehle, A. (2010) Comparison of local measures of spike time irregularity and relating variability to firing rate in motor cortical neurons. J. Comput. Neurosci. 29, 351-365

Raman, B., Joseph, J., Tang, J., Stopfer, M. (2010) Temporally diverse firing patterns in olfactory receptor neurons underlie spatiotemporal neural codes for odors. J. Neurosci. 30, 1994-2006

Riffell, J.A., Abrell, L., Hildebrand, J.G. (2008) Physical processes and real-time chemical measurement of the insect olfactory environment. J. Chem. Ecol. 34, $837-853$

Rinberg, D., Koulakov, A., Gelperin, A. (2006) Sparse odor coding in awake behaving mice. J. Neurosci. 26, 8857-8865

Rössler, W., Zube, C. (2011) Dual olfactory pathway in Hymenoptera: evolutionary insights from comparative studies. Arthropod Structure \& Development 40, 349-357

Rybak, J., Kuss, A., Lamecker, H., Zachow, S., Hege, H. C., Lienhard, M., Singer, J., Neubert, K., Menzel, R. (2010) The digital bee brain: integrating and managing neurons in a common $3 \mathrm{D}$ reference system. Front. Neuroinf. 4, 30

Rybak J (2012) The digital honey bee brain atlas. In: Galizia CG, Eisenhardt D, Giurfa M (Eds.). Honeybee Neurobiology and Behavior. Springer, Heidelberg, pp. 125-140

Rybak J, Menzel R (2010) Mushroom body of the honeybee. In Gordon M. Shepard, Sten Grillner
(Eds). Handbook of Brain Microcircuits. Oxford University Press, Oxford, pp. 433-38

Sachse, S., Galizia, C.G. (2002) Role of inhibition for temporal and spatial odor representation in olfactory output neurons: a calcium imaging study. J. Neurophysiol. 87, 1106-17

Sachse, S., Galizia, C.G. (2003) The coding of odourintensity in the honeybee antennal lobe: local computation optimizes odour computation. Eur. J. Neurosci. 18, 2119-2132

Sandoz, J.C. (2011) Behavioral and neurophysiological study of olfactory perception and learning in honeybees. Front. Syst. Neurosci. 5, 98. doi:10.3389/ fnsys.2011.00098

Schlief ML, Wilson RI (2007) Olfactory processing and behavior downstream from highly selective receptor neurons. Nat Neurosci 10: 623-630. doi:10.1038/ nn1881

Schmuker, M., Yamagata, N., Nawrot, M.P., Menzel, R. (2011) Parallel representation of stimulus identity and intensity in a dual pathway model inspired by the olfactory system of the honeybee. Front. Neuroeng. 4, 17. doi:10.3389/fneng.2011.00017

Shang, Y., Claridge-Chang, A., Sjulson, L., Pypaert, M., Miesenboeck, G. (2007) Excitatory local circuits and their implications for olfactory processing in the fly antennal lobe. Cell 128, 601-612

Silbering, A.F., Galizia, C.G. (2007) Processing of odor mixtures in the drosophila antennal lobe reveals both global inhibition and glomerulus-specific interactions. J. Neurosci. 27, 11966-11977

Sivan, E., Kopell, N. (2006) Oscillations and slow patterning in the antennal lobe. J. Comput. Neurosci. 20, 85-96

Smear, M., Shusterman, R., O'Connor, R., Bozza, T., Rinberg, D. (2011) Perception of sniff phase in mouse olfaction. Nature 479, 397-400. doi:10.1038/ nature 10521

Spors, H., Wachowiak, M., Cohen, L.B., Friedrich, R.W. (2006) Temporal dynamics and latency patterns of receptor neuron input to the olfactory bulb. J. Neurosci. 26(4), 1247-59

Staudacher, E.M., Huetteroth, W., Schachtner, J., Daly, K.C. (2009) A 4-dimensional representation of antennal lobe output based on an ensemble of characterized projection neurons. J. Neurosci. Meth. 180, 208-223

Stopfer, M., Bhagavan, S., Smith, B.H., Laurent, G. (1997) Impaired odour discrimination on desynchronization of odour-encoding neural assemblies. Nature 390, 70-4

Stopfer, M., Jayaraman, V., Laurent, G. (2003) Intensity versus identity coding in an olfactory system. Neuron 39(6), 991-1004

Strube-Bloss, M., Nawrot, M.P., Menzel, R. (2011) Mushroom body output neurons encode odorreward associations. J. Neurosci. 31(8), 3129-3140 
Sun, X.-J., Fonta, C., Masson, C. (1993) Odour quality processing by bee antennal lobe interneurons. Chem. Senses 18, 355-377

Szyszka, P., Ditzen, M., Galkin, A., Galizia, G., Menzel, R. (2005) Sparsening and temporal sharpening of olfactory representations in the honeybee mushroom bodies. J. Neurophysiol. 94, 3303-3313

Szyszka, P., Galkin, A., Menzel, R. (2008) Associative and non-associative plasticity in Kenyon cells of the honeybee mushroom body. Front. Syst. Neurosci. 2, 3

Szyszka, P., Demmler, C., Oemisch, M., Sommer, L., Biergans, S., Birnbach, B., Silbering, A.F., Galizia, C.G. (2011) Mind the gap: olfactory trace conditioning in honeybees. J. Neurosci. 31, 7229-7239

Thorpe, S., Delorme, A., Van Rullen, R. (2001) Spikebased strategies for rapid processing. Neural Netw. 14, 715-725

Tripp, B., Eliasmith, C. (2010) Population models of temporal differentiation. Neural Comput. 22(3), 621-659

Uchida, N., Mainen, Z.F. (2003) Speed and accuracy of olfactory discrimination in the rat. Nat. Neurosci. 6, 1224-1229

Urban, N.N. (2002) Lateral inhibition in the olfactory bulb and in olfaction. Physiol. Behav. 77, 607-12

Vickers, N.J., Christensen, T.A., Baker, T.C., Hildebrand, J.G. (2001) Odour-plume dynamics influ- ence the brain's olfactory code. Nature 410, 466470

Wesson, D., Carey, R., Verhagen, J., Wachowiak, M. (2008) Rapid encoding and perception of novel odors in the rat. PLoS Biol. 6, e82

Wick, S.D., Wiechert, M.T., Friedrich, R.W., Riecke, H. (2010) Pattern orthogonalization via channel decorrelation by adaptive networks. J. Comput. Neurosci. 28, 29-45

Wilson, R.I., Laurent, G. (2005) Role of GABAergic inhibition in shaping odor-evoked spatiotemporal patterns in the Drosophila antennal lobe. J. Neurosci. 25, 9069-79

Wilson, R., Turner, G., Laurent, G. (2004) Transformation of olfactory representations in the Drosophila antennal lobe. Science 303, 366-370

Wright, G.A., Thomson, M.G., Smith, B.H. (2005) Odour concentration affects odour identity in honeybees. Proc Biol Sci 272, 2417-2422

Wright, G.A., Carlton, M., Smith, B.H. (2009) A honeybee's ability to learn, recognize, and discriminate odors depends upon odor sampling time and concentration. Behav. Neurosci. 123, 36-43

Yamagata, N., Schmuker, M., Szyszka, P., Mizunami, M., Menzel, R. (2009) Differential odor processing in two olfactory pathways in the honeybee. Front. Syst. Neurosci. 3, 16. doi:10.3389/neuro.06. 016.2009 